\title{
Quasi Static and Fatigue Properties of Long Carbon Fiber Reinforced Polyamide
}

\author{
Jan-Marc Tiemann (D, Javad Mola, Philipp Land, Thorsten Krumpholz, and Ulrich Krupp
}

Submitted: 19 April 2021 / Revised: 3 August 2021 / Accepted: 4 August 2021 / Published online: 3 September 2021

\begin{abstract}
In this experimental work, the quasi static and fatigue properties of a $40 \mathrm{wt}$ \% long carbon fiber reinforced partially aromatic polyamide (Grivory GCL-4H) were investigated. For this purpose, microstructural parameter variations in the form of different thicknesses and different removal directions from injectionmolded plates were evaluated. Mechanical properties decreased by increasing misalignment away from the melt flow direction. By changing the specimen thickness, no change in the general fiber distribution pattern transversal and normal to the axis of melt flow was observed. It has shown that with increasing specimen thickness the quasi static properties along the melt flow direction decreased and vice versa resulting in superior properties normal to the melt flow axis. At around $5 \mathrm{~mm}$, an intersection suggests quasi-isotropic behavior. In addition, the fatigue strength of the material was significantly higher in the flow direction than normal to the flow direction. No change in fatigue life was observed while changing specimen thickness. The Basquin equation seems to describe the effect of stress amplitude on the fatigue strength of this composite. Scanning electron microscopy was used to investigate fracture surfaces of tested specimens. Results show that mechanical properties and morphological structures depend highly on fiber orientation.
\end{abstract}

$\begin{array}{ll}\text { Keywords } & \begin{array}{l}\text { damage behavior, electron microscopy, fatigue, } \\ \text { fatigue testing, long fiber reinforced plastics, } \\ \text { microstructure, quasi static properties }\end{array}\end{array}$

\section{Introduction}

Long fiber reinforced thermoplastics (LFT) are being used in a variety of industry sectors due to their good combination of processability and high specific mechanical properties (Ref 1). Especially in the automotive industry, materials such as metals and thermoset plastics are being substituted more frequently by LFTs. LFTs allow the reduction of both, costs and vehicle weight. Past developments have shown that thermoplastic composites market have seen high consumption growth rates in the plastic industry with an ongoing projected growth from USD 22.2 billion in 2020 to USD 31.8 billion by 2025, at a CAGR of $7.5 \%$ (Ref 2). The possibility of injection molding lowers the time of production in combination with minimal finishing procedures. Additionally, injection molding allows the suppliers to integrate parts, which leads to a substantial cut in the overall assembly costs. Furthermore, their intrinsic recyclability is being recognized as a strong driving force for further applications (Ref 3). Mechanical properties of short fiber reinforced thermoplastics (SFT) have been already assessed by a variety of authors in terms of fiber orientation and different loading conditions (Ref 4, 5). Compared to SFTs, LFTs consist

Jan-Marc Tiemann and Javad Mola, Laboratory of Materials Design and Structural Integrity, University of Applied Science Osnabrück, Osnabrück, Germany; Philipp Land and Thorsten Krumpholz, Laboratory of Plastics Technology, University of Applied Science Osnabrück, Osnabrück, Germany; and Ulrich Krupp, Steel Institute, RWTH Aachen University, Aachen, Germany. Contact e-mail: janmarc.tiemann@hs-osnabrueck.de. of long reinforcement fibers, such as carbon, glass, aramid or natural fibers, with initial lengths larger than $1 \mathrm{~mm}$. During processing, the embedded fibers build a dense fiber network improving the overall mechanical and thermal properties. Thus, general advantages of LFTs are excellent flexural, tensile and impact properties, low creep rates, low shrinkage, high fatigue strengths and a low moisture absorption for hygroscopic matrix materials (Ref 6). In spite of the advantages of LFTs, there is still a lack in correlating the mechanical performance with the evolution of fiber orientation during injection molding. The prevalent fiber orientation of injection-molded LFTs is mainly affected by the thickness of the specimen and the removal direction of specimens from the plate. Due to the increasing use of LFTs for structurally loaded components, it is essential to characterize and further predict the composite behavior under quasi static and cyclic loading conditions. Fatigue tests are costly and time-consuming. The ability to describe the behavior reduces costs and leads to clear guidelines for engineering design. However, due to an intrinsic anisotropic behavior of injection-molded LFTs, specimens for mechanical testing in one particular direction are not sufficient to describe the mechanical properties comprehensively. In such cases, the extent of anisotropy is mainly affected by the fiber orientation distribution and thickness of the specimen. Bernasconi et al. (Ref 7) verified that extraction direction of specimens from injection-molded plates influenced the properties of short glass fiber reinforced Polyamide 6. The Young's modulus, ultimate tensile strength (UTS) and fatigue strength exhibited a decrease with increasing extraction angle with reference to the melt flow direction. Similar results were obtained by Zhou et al. (Ref 5) and Brunbauer (Ref 6), where the best mechanical performance was observed parallel to the melt flow direction in which the fibers were extending. Increasing misorientation angles led to a distinct decrease in quasi static and fatigue properties. In the latter case, the specimens exhibited a typical skin-core layer structure with fibers being aligned with melt flow directions in the peripheral areas of the part and perpendicular to it in the 
core regions. Figure 1 illustrates a typical volume element of an injection-molded plate with melt flow direction highlighted on top. Comparing SFTs with LFTs, the idealized model illustrated in Fig. 1 is well suited for SFTs recognizable by a clear transition from the shell to the core layers. In LFTs, long initial fiber lengths and high fiber volume fractions lead to fiber-fiber interactions generating dense fiber networks and thus morphologies exhibiting high looped and interconnected fibers.

Relatively, few data have been published on the fatigue performance of LFTs. Grove et al. (Ref 8) investigated the fatigue performance of glass fiber reinforced thermoplastics with short and long glass fibers and found superior fatigue properties for long fiber reinforced composites, especially for high stress amplitudes. The beneficial effect of long fiber reinforcement exceeding the critical fiber length was primarily attributed to an additional fiber breakage mechanism not occurring in short fiber composites. Long glass fiber reinforced specimens milled from plates in parallel and transversal direction relative to the melt flow direction have been investigated by Goel et al. (Ref 9). They came to the result that the parallel specimens showed higher fatigue resistance because of a better load transfer by fibers aligned toward the loading direction. Similar to SFTs, LFTs are prone to failure due to hysteretic heating when testing at frequencies larger than $10 \mathrm{~Hz}$. Studies of Goel et al. and Bellenger et al. (Ref 9, 10) revealed that the higher the testing frequency, the lower is the fatigue life. In (Ref 10) was found: the extent of thermal and mechanical fracture modes depend on the frequency. For $10 \mathrm{~Hz}$, they concluded that both thermal and mechanical fracture occurred. At $2 \mathrm{~Hz}$, the matrix was completely in glassy state at break. Investigations on long glass fiber reinforced polypropylene tested at $10 \mathrm{~Hz}$ reached an equilibrium heat level at cyclic loading suggesting the absence of thermal fracture (Ref 9).

Mallick and Zhou (Ref 6) investigated the influence of stress ratio on the fatigue behavior of short glass fiber reinforced polyamide. Increasing the stress ratio, i.e., increasing the tensile mean stress, led to a reduction in fatigue life. Regarding the influence of stress ratio, it is often observed that an increase in stress ratio lowers the number of cycles to failure for a constant stress amplitude. Bondy et al. (Ref 11) investigated directly compounded long carbon fiber reinforced polyamide under tensile fatigue loading taken at $0^{\circ}, 45^{\circ}$ and $90^{\circ}$ with respect to flow. The results show decreasing composite fatigue peak stresses with increasing deviation from the melt flow direction. This property degradation was mainly reasoned by unfavorable

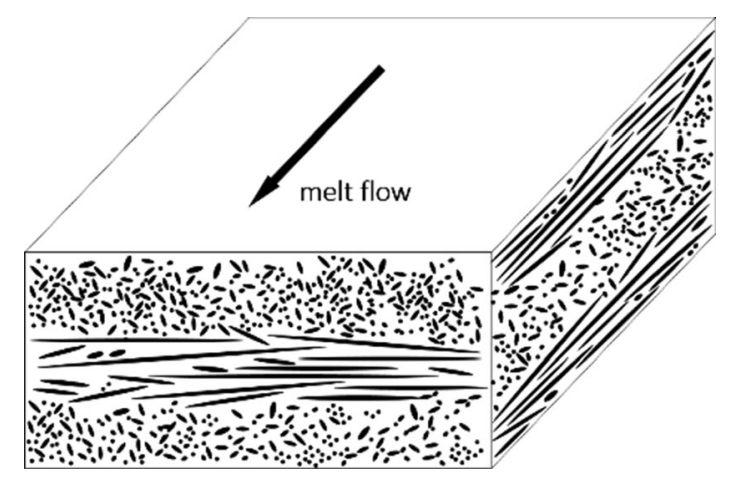

Fig. 1 Schematic illustration of the idealized skin-core layer structure induced by injection molding processing aligned fibers with respect to the applied load and dry and poorly impregnated fibers. To assess the above-mentioned parameters, quasi static and cyclic tests have been conducted using specimens taken out from unevenly thick plates at different directions relative to the melt flow. Fatigue tests were performed at different stress ratios including dwelling and alternating loading conditions. All mechanical tests have been accompanied by scanning electron microscopy to identify the failure characteristics with respect to the applied load.

\section{Experimental}

\subsection{Materials}

The investigated composite Grivory GCL-4H consists of a heat-stabilized semi-crystalline polyamide with a partially aromatic polyamide and a long carbon fiber reinforcement. The fiber-web structure created inside the component is responsible for a wide series of improved thermomechanical properties. To mention are a high stiffness and tensile strength, a high creep resistance and a high fatigue strength. The raw material was supplied by EMS Chemie AG, Domat/Ems, Switzerland and is in a pultruded condition, consisting of parallel strands of fibers embedded in a thermoplastic matrix. Compared to extrusion-compounded pellets, the initial fiber length is constant at $10 \mathrm{~mm}$, ensuring maximal fiber length after further processing. Figure 2 illustrates an $\mathrm{x}$-ray micro tomography image of a pultruded long carbon fiber reinforced pellet. The parallel oriented carbon fibers (white) within the polyamide matrix (black) can be clearly seen. This examination was performed in order to ensure unbroken and uniformly aligned fibers in the pellet. Furthermore, the initial fiber length of $10 \mathrm{~mm}$ was confirmed.

The mean diameter of the carbon fibers is $10 \mu \mathrm{m}$. All fibers have been treated with a proprietary sizing to improve fiber matrix adhesion properties. Mass fraction and volume fraction of the fibers are 40 and $30 \%$, respectively. According to the material data sheet provided by the EMS Chemie AG the Young's modulus and the tensile strength of the investigated material amounts to $28.5 \mathrm{GPa}$ and $300 \mathrm{MPa}$, respectively.

\subsection{Manufacturing Process}

All specimens were manufactured by injection molding and supplied by EMS Chemie AG, Domat/Ems. Beside the raw pellet characteristics, fiber length is strongly affected by the processing parameters during injection molding. Due to

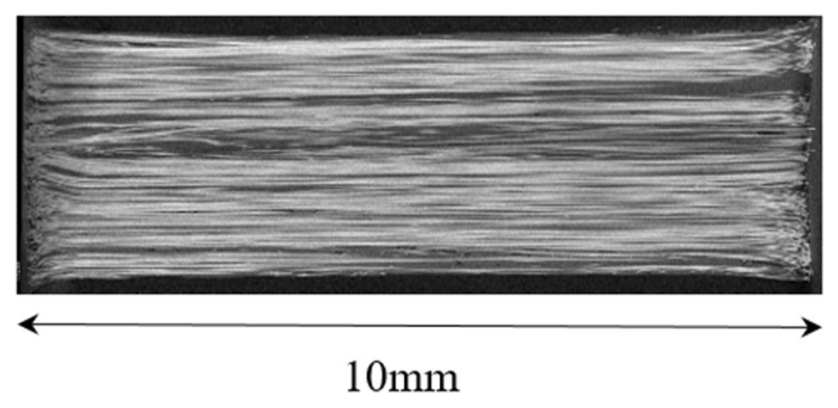

Fig. 2 X-ray micro tomography of a pultruded PA+CF40 pellet of 40 wt.\% (30 vol.\%) carbon fiber reinforced (PA6T,6I + PA6,6) matrix 
predominating conditions such as changing shear forces and velocity gradients, the initial fiber length of $10 \mathrm{~mm}$ undergoes a significant decrease during subsequent processing (Ref 12, 13). Especially low injection speeds and peripherical screw speeds proved to have an impact on the material properties. The degree of fiber length shortening was quantified by determining the average fiber length in the injection-molded $4 \mathrm{~mm}$ plate. For this purpose, the plates were placed in a muffle furnace at $550{ }^{\circ} \mathrm{C}$ for $30 \mathrm{~min}$ to allow for the decomposition of the thermoplastic matrix. What is left over is a dense network of interconnected fibers. Based on light optical microscopic (LOM) examinations using extracted samples $15 \times 15 \mathrm{~mm}$ in size, the arithmetic mean fiber length $L_{\mathrm{n}}$ and the weighted mean fiber length $L_{\mathrm{w}}$ were calculated using Eq (1) and (2):

$L_{n}=\frac{\sum_{i=1}^{n} L_{i}}{n}$,

$L_{w}=\frac{\sum_{i=1}^{n} n_{i} \cdot L_{i}^{2}}{\sum_{i=1}^{n} n_{i} \cdot L_{i}}$,

$L_{i}$ is the length of the $i$-th fiber in the sample and $n_{i}$ is the sample frequency with the length increment range $l_{i+1}$ to $l_{i}$. The arithmetic average is always smaller than the weighted average and is additionally strongly influenced by the amount of fibers and fragments present. The weighted average, in contrast, is predominantly influenced by the fraction of long fibers present. Weighted average is a more meaningful parameter for correlation with the mechanical behavior, since mechanical properties are strongly influenced by the volume of fibers of a given length rather than by the number of fibers (Ref 14). For the quantification of $L_{\mathrm{n}}$ and $L_{\mathrm{w}}$, more than 10000 fibers were counted.

One of the problems associated with the fragmentation of fibers during injection molding is the generation of new fiber surfaces, in particular fiber ends not coated with the coupling agent, leading to mechanical properties lower than the achievable maximum. Here, the most-affected properties are the tensile strength and impact toughness (Ref 15). To avoid a deterioration in mechanical properties, the injection molding parameters were designed based on preliminary statistical tests targeting for long fibers and high mechanical properties by varying processing parameters.

\subsection{Testing Methods}

All tests were performed in the conditioned state. To assess the influence of fiber orientation distribution for both, tensile and fatigue tests, large standardized dumbbell specimens of the type 1A (Fig. 3a) as well as small standardized dumbbell specimens of the type 1BA water jet cut from the plates (Fig. 3b), according to DIN EN ISO 527-2, were tested. In the further course of the work, the designations $\mathrm{LS}$ and $\mathrm{SSx}^{\circ}$ are used to refer to large and small specimens, respectively. In the latter case, $x^{\circ}$ refers to the angle of the specimen axis relative to the axis of melt flow. LSs were directly produced by injection molding without finishing procedures. The parallel length is $80 \mathrm{~mm}$ and the thickness amounts to $4 \mathrm{~mm}$. SSs are water jet cut from $4 \mathrm{~mm}$ thick plates at different angles to the melt flow axis (Fig. 3b). In order to achieve comparability between all specimens, the extraction locations were chosen far from the injection gate and end-filling zones. Tested angles are $0^{\circ}, 30^{\circ}$, $45^{\circ}, 60^{\circ}$ and $90^{\circ}$. Furthermore, tested plate thicknesses for

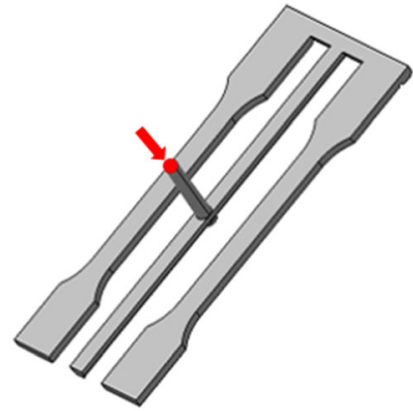

(a)

(b)

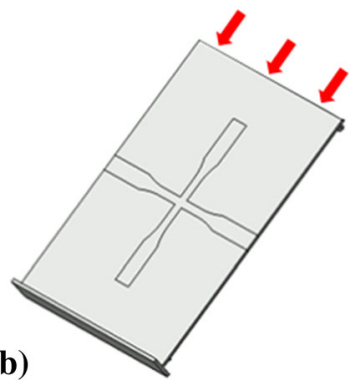

Fig. 3 Schematic representation of the specimen geometry within the injection-molded parts: (a) large standardized dumbbell sample (LS) and (b) water jet cut sample from a plate (SS). As an illustration just the removal directions $0^{\circ}$ and $90^{\circ}$ are highlighted. The red arrows mark the injection direction

tensile test specimens amount to 2, 4, 6, 8 and $10 \mathrm{~mm}$. All tests were done in a servo hydraulic testing machine with a $25 \mathrm{kN}$ load cell. Tensile properties were determined at a constant crosshead displacement speed of $1 \mathrm{~mm} / \mathrm{min}$ until fracture occurs. Strain measurement was done using a clip-on extensometer with an initial opening of $25 \mathrm{~mm}$. For standardized testing specimens $1 \mathrm{~A}$ and 1BA the tensile modulus was determined according to the DIN EN ISO 527-1 standard.

Fatigue properties of polymers depend strongly on the applied frequency, stress amplitude and viscoelastic behavior of the composite itself. In the case of high frequencies, hysteretic heating is the main failure mechanism dominated by viscoelastic matrix properties as well as a low thermal conduction of the matrix. The corresponding increase in temperature can lead to a decrease of the composite Young's modulus, leading to premature failure. Preliminary tests at $10 \mathrm{~Hz}$ confirmed the absence of hysteretic heating. Sudden temperature peaks due to localized deformation only play a role shortly before fracture. Fatigue tests were performed at stress ratios of $R=0.1,0.2,0.5$ and -1 . Since mean stress $\sigma_{\mathrm{m}}$ is equal to $0.5 \sigma_{\max }(1+R)$, the $R$ ratio variation corresponds to $\sigma_{\mathrm{m}}$ values of $55,60,75$ and $0 \%$ of $\sigma_{\max }$. Due to the anisotropy, cyclic tests were carried out for samples exhibiting angles of $0^{\circ}, 30^{\circ}, 45^{\circ}, 60^{\circ}$ and $90^{\circ}$ relative to the melt flow direction for $4 \mathrm{~mm}$ thick samples. The influence of specimen thickness was investigated using small dumbbell specimens with thicknesses of $2 \mathrm{~mm}$ and $4 \mathrm{~mm}$ taken parallel and perpendicular to the axis of melt flow.

For each stress level, at least three samples were tested. The test results were plotted in a S-N diagram, allowing to derive the parameters of Basquin's Eq (3) (Ref 16):

$\frac{\Delta \sigma}{2}=\sigma_{f}^{\prime}\left(2 N_{f}\right)^{b}$

, where $\frac{\Delta \sigma}{2}$ is the stress amplitude, $\sigma_{f}^{\prime}$ is the fatigue strength coefficient, $N_{\mathrm{f}}$ is the number of cycles to failure, $2 N_{\mathrm{f}}$ the number of load reversals and $b$ the fatigue strength exponent, a quantitative measure of the fatigue sensitivity. A larger absolute value of $b$ implies a higher slope of the $\log \Delta \sigma / 2$ vs. $\log N_{\mathrm{f}}$ curve, i.e., a pronounced decrease of the fatigue strength when shifting to higher numbers of load cycles. It is generally considered for ductile engineering materials that values of $b$ range from -0.05 to -0.12 (Ref 17 ). Taking existing fatigue studies from the literature into account, it seems that conven- 
tional composites do not show a fatigue limit, damage progresses are active throughout the entire life of the material which causes failure even at very low applied stresses (Ref 18). Therefore, no criteria for an endurance limit was set.

\subsection{Microstructural and Damage Investigation}

To investigate the effect of processing parameters on the morphology, cross sections of injection-molded specimens were prepared for materialographic examinations. In cases where the plate thickness was varied, it is expected that the morphological development in the thickness direction is influenced by the prevalent cooling and melt flow conditions. Cross sections were examined with a Keyence VHX 5000 digital light optical microscope (LOM). To obtain deeper microstructural insights, 2D panorama micrographs of each cross section were taken. Sample preparation was performed by using an automatic polisher; the preparation process consisted of grinding (down to $\mathrm{SiC}$ grit size 320) for $1 \mathrm{~min}$ followed by polishing using diamond suspensions in the sequence $9 \mu \mathrm{m}$ for $15 \mathrm{~min}, 3 \mu \mathrm{m}$ for $10 \mathrm{~min}$, and $1 \mu \mathrm{m}$ for $5 \mathrm{~min}$.

The fracture surfaces of the fatigued specimens were examined using a high-resolution Zeiss Auriga scanning electron microscope (SEM). Image acquisition was adjusted for a proper topography contrast. Fracture surfaces were initially ultrasonically cleaned and then sputter-coated with $\mathrm{Pt} / \mathrm{Ir}$ to improve electrical conductivity at surface.

\section{Results and Discussion}

\subsection{Processing-Induced Morphology}

Microstructural examination results after injection molding are illustrated in Fig. 4. After injection molding, the investi- gated material exhibited a three-layered microstructure consisting of a core layer surrounded by two shell layers. Examinations at higher magnification revealed that the fiber orientation pattern was different within the layers. Whereas the shell region is characterized by fibers oriented in the melt flow direction, the predominant fiber orientation in the core region can be described as $45-90^{\circ}$ to the melt flow direction. Due to a high interconnection between the fibers, no clear transition between the layers was observed. The relative thickness of the three layers was found to vary with the plate thicknesses. The shell layer thickness is almost constant at around $1 \mathrm{~mm}$, while the width of the core region increases with increasing specimen thickness Fig. 4(c), (e). Investigations of (Ref 19) point out that a larger extent of the core region changes the overall mechanical properties by generating unfavorable fiber orientation distributions in the specimen center ( Ref 19). The strength profile from the surface to the core of the specimens depends strongly on the fiber orientation distribution of the specimen. Generally, thinner specimens exhibit higher strength due to their high amount of fibers in the shell layers orientated in parallel to the loading axis. Consequently, a distinction between thin and thick specimens has to be done since the strength profiles are essentially different. Thin samples can be characterized by high fractions of MFD-aligned fibers in the shell layer with a less influenced core layer. In the core layer primarily planar fiber distributions were observed. In contrast, specimens with thicknesses of $5 \mathrm{~mm}$ and more did not show a higher strength. Here, the high amount of fibers in the core layer were proven not to be parallel to any surface of the molding and consequently not adding additional strength in any direction. The conclusions of (Ref 19) were confirmed by comparing the core regions of the light optical micrographs in Fig. 4(b), (c).

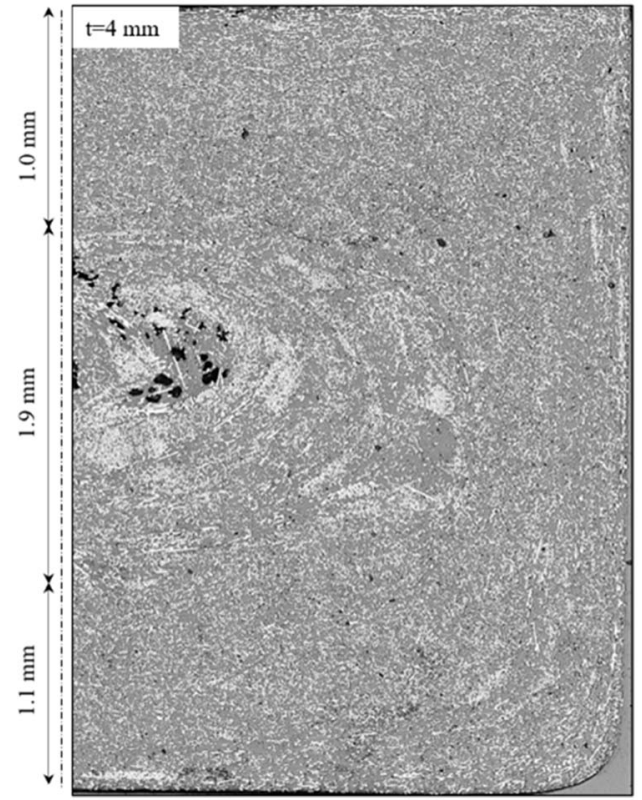

(a)

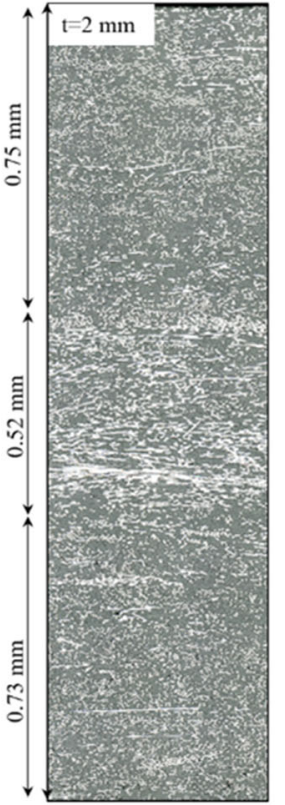

(b)

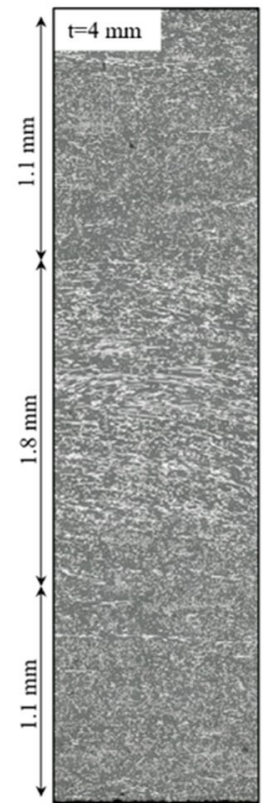

(c)

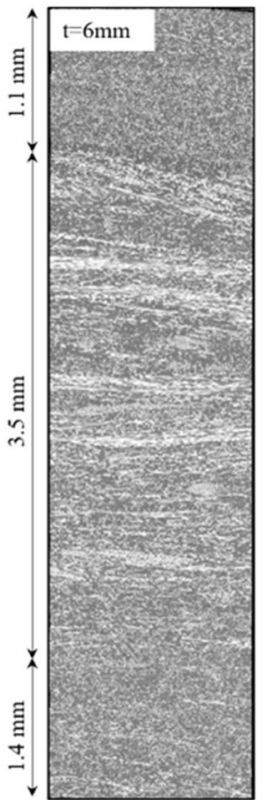

(d)

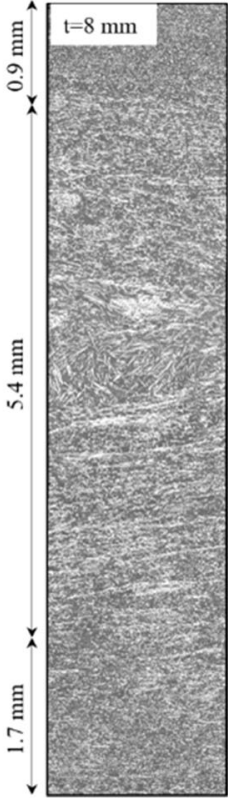

(e)

Melt flow direction

Fig. 4 Light optical micrographs of sections normal to the melt flow direction (MFD) for (a) directly molded large dumbbell specimens with vacuum voids in the center and small dumbbell specimens cut from plates with thicknesses of (b) $2 \mathrm{~mm}$, (c) $4 \mathrm{~mm}$, (d) $6 \mathrm{~mm}$ and (e) $8 \mathrm{~mm}$ 
The illustrated micrographs serve as representative images for the respective thicknesses and cover mean layer thickness values. The determination of the transition zone from shell to skin layer was done by judging the highest difference in major fiber orientation distribution. For most cases, this limit can be clearly defined. Considering constant cooling conditions during processing the outer specimen volume undergoes the same temperature gradient independently of the extent of the inner volume. Thus, the shear forces at the outside of the specimens can be assumed to be constant resulting in evenly thick skin layers for all plates. In contrast, fiber alignment with the melt flow direction is very weak in central regions of all plates. It has to be mentioned that for the sake of clarity, the cross section of LS is reduced to half. Only for the directly injected LS, vacuum voids were observed in the core regions, Fig. 4(a). It was noticeable that vacuum voids primarily develop in fiber rich areas in the center. Such voids were not observed in the milled SSs. Since the entire cross section of the LS was in contact with the mold, its core area is completely surrounded by a shell layer. This was not the case for the SSs as they were milled from plates. Consequently, the overall fraction of fibers aligned toward the melt flow was highest for LS. However, comparing the specimen geometries SS is representing the reality better by negligible impacts from side faces often not being existing in real components.

As mentioned earlier, the initial fiber length of $10 \mathrm{~mm}$ undergoes significant shortening during manufacturing. This happens firstly during the plasticizing process and secondly while injecting into the mold. The extent of fiber breakdown can be demonstrated by comparing the prevalent fiber length in the melt strand and the final specimen. Statistical analyses of the average fiber lengths indicated that the arithmetic mean fiber length $\mathrm{L}_{\mathrm{n}}$ and the weighted mean fiber length $L_{\mathrm{w}}$ were $L_{\mathrm{n}}=1038 L_{\mathrm{w}}=2462 \mu \mathrm{m}$ for the melt strand and $L_{\mathrm{n}}=1267$ and $L_{\mathrm{w}}=1726 \mu \mathrm{m}$ in the plate, respectively. For this purpose, more than 10000 fibers taken from different positions of the plates were used. Considering this, it is visible that there is a significant fiber length degradation during plasticizing and an additional shortening when flowing into the cooled mold.

Figure 5 illustrates light optical micrographs of the shell and core layers as a function of the removal direction from a $4 \mathrm{~mm}$ plate. For clarity reasons, only sections with normal vectors making angles of $0^{\circ}, 45^{\circ}$ and $90^{\circ}$ with the melt flow direction are shown in Fig. 5. As the latter angle increases, the shape of the fibers in the shell layer changes from circular to elliptical. This indicates a strong fiber alignment with the injection axis in the shell layer. This observation is in good agreement with the results from Bernasconi et al. (Ref 7). The morphology in the core area is more heterogeneous compared to the shell layer as the fibers point in various directions. Despite the larger scatter in the orientation of fibers in the core layer, the alignment of fibers is clearly opposite to that observed for the shell layer. With a large fraction of fiber aligned $45-90^{\circ}$ with respect to the melt flow direction, evidenced by the large fraction circular fibers in the transverse direction, the core layer is expected to make a larger contribution to the mechanical properties for higher misorientation angles.

\subsection{Quasi static Properties}

Figure 6(a), (b) summarize results of tensile tests, tensile strength and Young's modulus, respectively, as a function of the plate thickness. These values were measured using milled specimens aligned $0^{\circ}$ and $90^{\circ}$ with respect to the axis of the melt flow. Generally, a distinct anisotropic mechanical behavior appears. Nearly the highest tensile strength and stiffness were measured for $\mathrm{SS}^{\circ}$ with a thickness of $2 \mathrm{~mm}$ reasoned by the highest fraction of shell layer relative to whole cross section area. Thus, the $2 \mathrm{~mm}$ samples also proved to have the highest degree of anisotropy. Except for an increase of the $4 \mathrm{~mm}$ samples for both, tensile and flexural properties, a decrease longitudinal to the MFD can be observed followed by a plateau. The mechanical behavior for the transverse removal direction behaves odd, exhibiting rather advancing properties with increasing plate thickness. Regarding the layer characteristics for the $4 \mathrm{~mm}$ specimens, the overall layer thicknesses for the core and shell layer amount to 1.8 and $2.2 \mathrm{~mm}$, respectively. In the case of $6 \mathrm{~mm}$ samples, the sizes are $3.5 \mathrm{~mm}$ and $2.5 \mathrm{~mm}$ for the core and shell layer, respectively. This development is already suggesting a more beneficial role of the core region providing more fibers pointing toward the loading axis. Whereas the Young's modulus is constantly increasing, the tensile strength is reaching its transverse in the range of 4$6 \mathrm{~mm}$. Constantly improving flexural properties can be related to an additional contribution of inside fibers being aligned longitudinal as well as less fiber length degradation during processing because of a larger mold cross section. Considering this development, it can be concluded that with decreasing shell layer fractions strength and flexural properties parallel to MFD decrease and vice versa. Interestingly, this trend does not hold true for the tensile strength for thickness values of $6 \mathrm{~mm}$ and higher. According to the previously mentioned investigations of Horst et al. (Ref 19), it can be assumed that the higher the fraction of the core volume the more potential damage sites are provided and more fibers point in directions not favoring the longitudinal or transverse loading direction. Furthermore, when having a look on the fracture surface appearance of the 8 and $10 \mathrm{~mm}$ samples, the inner volume often appears similar to a solidified polymer melt front during the filling process, suggesting this microstructural arrangement to be weakest.

At approximately $5 \mathrm{~mm}$, there is a crossing point of the $0^{\circ}$ and $90^{\circ}$ curves. This intercept suggests a quasi-isotropic behavior at around $5 \mathrm{~mm}$ thickness. However, the dominance of the core layer is not as strong as the properties reached for values tested $0^{\circ}$ to MFD for $t=2$ and $4 \mathrm{~mm}$ plate thickness. In particular, the tensile properties exhibit significantly lower values. In contrast to the longitudinal samples, the Young's modulus perpendicular to the injection direction almost reach the highest values measured parallel to the melt flow axis.

The differences in the tensile properties between LS and SS milled at different directions from a $4 \mathrm{~mm}$ plate are summarized in Fig. 7. Both tensile and flexural properties show a decreasing tendency from LS exhibiting the highest value. These results might be attributed to different strain distributions due to variations of the local apparent elastic properties along the specimen section, which in turn are closely related to changes in fiber orientation occurring. Parallel to the mold flow direction $\left(0^{\circ}\right.$ angle $)$, the fraction of fibers aligned in the loading direction is maximal, resulting in a superior tensile strength. As the angle deviation from the MFD increases, the tensile strength as well as the stiffness decreases.

LS were tested for comparison. In this case, the mechanical properties are highest. It can be observed that the higher the fraction of fibers oriented in loading direction, the better is the mechanical performance. Conversely, plasticity occurs early for transverse specimens where the fraction of MFD oriented fibers 


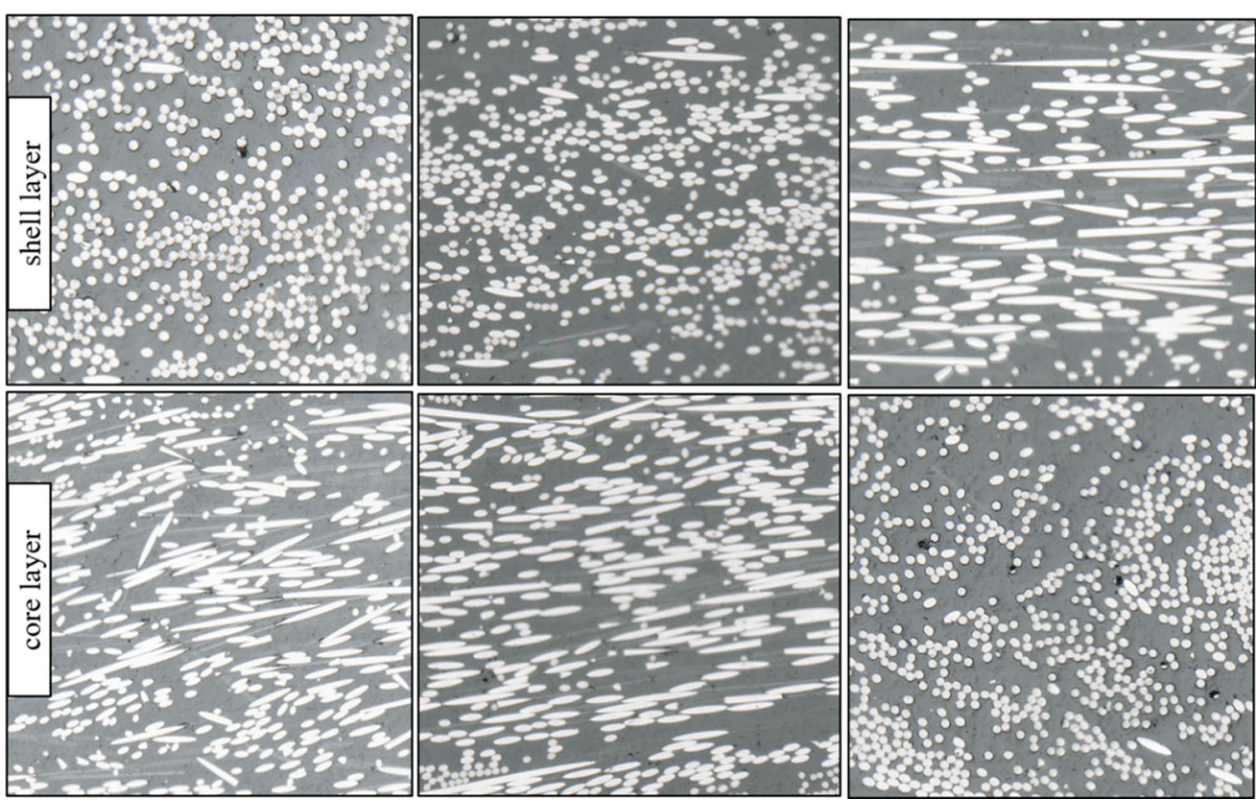

Fig. 5 Light optical micrographs showing the structure of the shell and core region of a $4 \mathrm{~mm}$ plate as a function of the sample direction. Angles on top indicate directions between the normal vector of the plane of polish and the melt flow direction
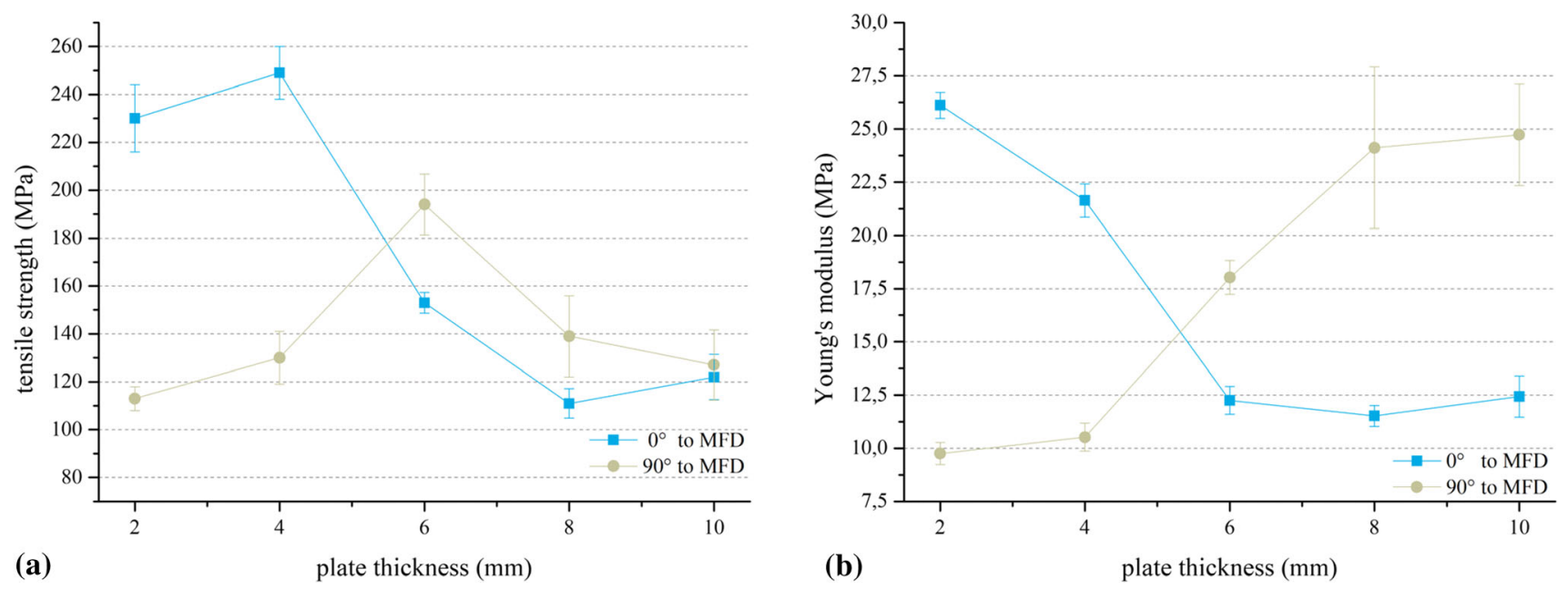

Fig. 6 (a) Tensile strength and (b) Young's modulus for specimens parallel and transversal to the melt flow directions taken from plates with different thicknesses

is less and thus the stiffness is low. The strength of the LS is higher in contrast to $\mathrm{SS} 0^{\circ}$ despite of having voids in the central regions. Brunbauer et al. obtained similar results when testing standardized dog bone specimens against small specimens milled from plates (Ref 6). Consequently, it can be concluded that material characterization on directly injected standardized specimens provide overestimated values of the reality of plates and complex mechanical components.

\subsection{Fatigue Properties}

All fatigue data were analyzed by the classical stress-life approach according to ASTM E 739-91 (Ref 19). In the diagrams illustrated in Fig. 8, data are drawn in terms of stress amplitude $\Delta \sigma / 2$ versus the number of cycles to failure $N_{\mathrm{f}}$ in a double logarithmic way.
Fatigue tests at different stress ratios yield the result that fully reversed loading conditions lead to the best fatigue performance. As it can be seen in the S-N curves in Fig. 8, an increasing mean stress leads to a pronounced decrease in the fatigue strength. Comparing $R=-1$ and $R=0.1$, for life time periods exceeding $2 \times 10^{6}$ cycles (often used as fatigue limit criterion of plain carbon steel) corresponding stress amplitudes of $\Delta \sigma / 2=81 \mathrm{MPa}$ for $R=-1$ and $\Delta \sigma / 2=68 \mathrm{MPa}$ for $R=0.1$ were measured. Between $2 \times 10^{4}<N_{\mathrm{f}}<2 \times 10^{6}$ cycles the approach of Basquin proved to be well fitting (Ref 16). Calculated values for the fatigue strength coefficient $\sigma_{\mathrm{f}}^{\prime}$ and fatigue strength exponent $\mathrm{b}$ are illustrated in Table 1 . The material fatigue strength exponent $b$ is important in reflecting the slope of the $\mathrm{S} / \mathrm{N}$ curves. The values of fatigue strength exponent were found to be $b=-0.03$ and $b=-0.05$, respectively. The investigations revealed lower $b$ values for 

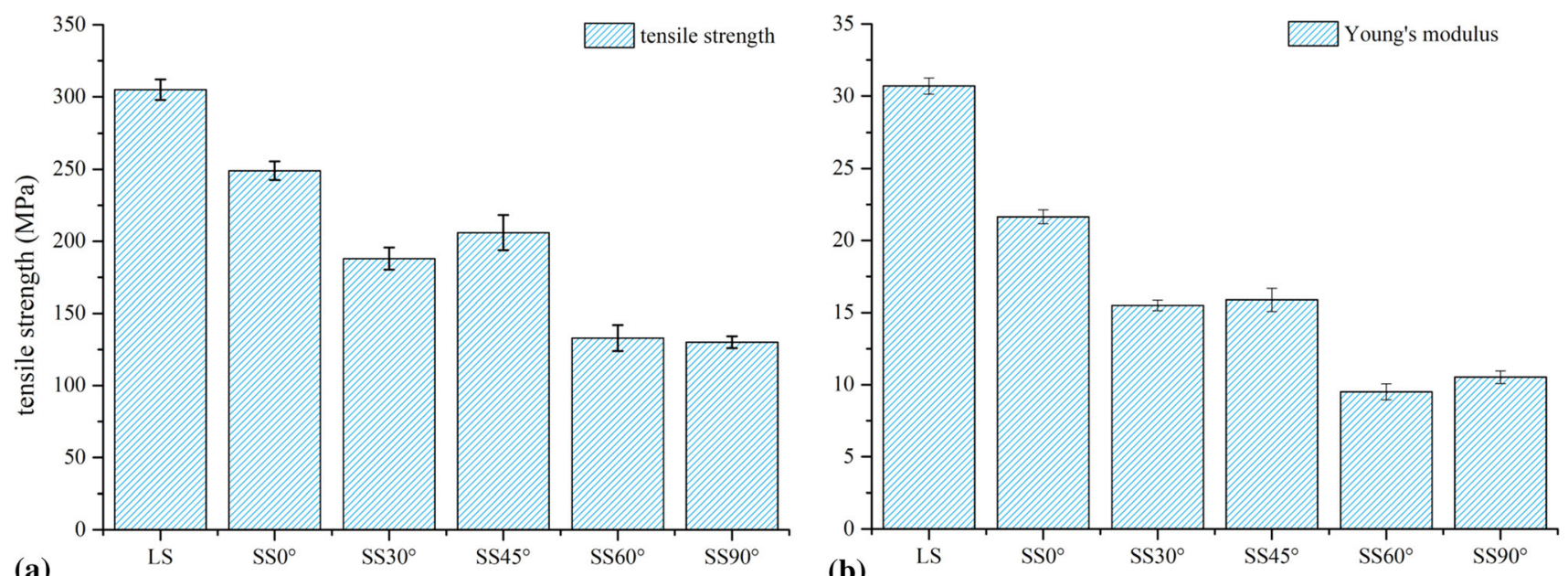

Fig. 7 (a) Tensile strength and (b) Young's modulus for LS compared to specimens taken from different directions of a plate 4 mm in thickness

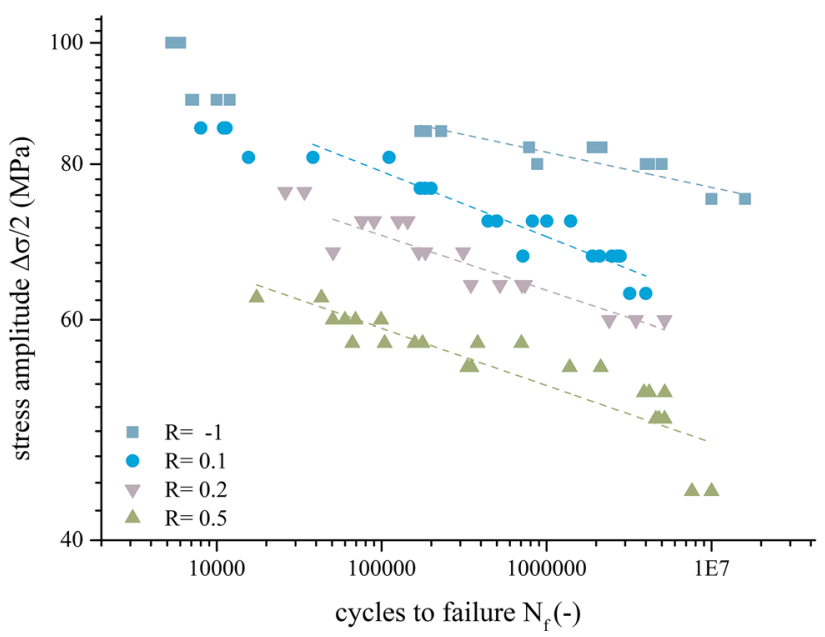

Fig. 8 Influence of different stress ratios $R$ on the fatigue properties tested at LS. The Wöhler lines for each stress ratio are shown dashed. Fatigue testing parameters are $f=10 \mathrm{~Hz}$ and $T=R T$

Table 1 Fatigue strength coefficients $\sigma_{\mathrm{f}}^{\prime}$ and fatigue strength exponents b

\begin{tabular}{lccrr}
\hline Thickness & Removal direction & Stress ratio $\boldsymbol{R}$ & $\boldsymbol{b}$ & $\boldsymbol{\sigma}_{\mathbf{f}}{ }^{\prime}$ \\
\hline \multirow{4}{*}{$4 \mathrm{~mm}$} & LS & -1 & -0.028 & 123 \\
& & 0.1 & -0.051 & 143 \\
& & 0.2 & -0.044 & 116 \\
$4 \mathrm{~mm}$ & & 0.5 & -0.046 & 100 \\
& $0^{\circ}$ & 0.1 & -0.053 & 117 \\
& $30^{\circ}$ & & -0.033 & 64 \\
& $45^{\circ}$ & & -0.032 & 51 \\
& $60^{\circ}$ & & -0.034 & 55 \\
$2 \mathrm{~mm}$ & $90^{\circ}$ & & -0.057 & 62 \\
& $0^{\circ}$ & 0.1 & -0.058 & 128 \\
& $90^{\circ}$ & & -0.073 & 68 \\
\hline
\end{tabular}

increasing stress ratio values indicating a slower damage evolution during cyclic loading. As can be observed from Fig. 9(a), the influence of fiber orientation distribution for 2 and
$4 \mathrm{~mm}$ small standardized specimens is investigated. While quasi static tests revealed a different behavior for 2 and $4 \mathrm{~mm}$ samples, the S-N-curves are nearly the same. Calculated values for the fatigue strength exponents confirm these observations. For both thicknesses comparable $b$ values were obtained suggesting a similar damage propagation rate for the respective direction. The reduction of the expected fatigue performance from the $2 \mathrm{~mm}$ samples can be explained including the microscopic images of the microstructure (Fig. 4b). In the transition zone from the shell to the core layer, voids were observed locally weakening the microstructure. Since damage during fatigue sets in locally, defects in the microstructure are more detrimental with respect to the fatigue properties as compared to the static properties. As a result, the expected superior fatigue performance of $2 \mathrm{~mm}$ samples could not be confirmed.

The effect of fiber orientation distribution with reference to of melt flow direction is illustrated in Fig. 9(b). By including the stiffness values from quasi static testing results in Fig. 7(b) highest fatigue properties are estimated for $\mathrm{SS}^{\circ}$ specimens. Angles from $30^{\circ}$ to $90^{\circ}$ are expected to show similar fatigue properties due to similar stiffness's.

The $\mathrm{S} / \mathrm{N}$ diagrams in Fig. 9 indicate that the fatigue performance of the $\mathrm{SSx}^{\circ}$ specimens is very sensitive upon the loading axis relative to the axis of the melt flow. The highest fatigue resistance was obtained for $\mathrm{SS} 0^{\circ}$. Deviation angles apart from $0^{\circ}$ show significantly lower fatigue strengths. Already $30^{\circ}$ difference apart from the parallel direction lead to a decrease of approximately $45 \%$ in fatigue life. Angles of $45^{\circ}, 60^{\circ}$, and $90^{\circ}$ revealed similar fatigue performances. Contrary to the assumption of similar fatigue properties of $\mathrm{SS} 30^{\circ}, \mathrm{SS} 45^{\circ}, \mathrm{SS} 60^{\circ}$, and $\mathrm{SS} 90^{\circ}$, the results of $\mathrm{SS} 30^{\circ}$ show surprisingly higher fatigue strength than the other deviant angles from the axis of melt flow. Bernasconi et al. (Ref 7) investigated short fiberreinforced Polyamide 6 samples taken from different directions to the axis of melt flow. The results confirm superior properties for samples $0^{\circ}$ to the axis of melt flow, followed by $30^{\circ}$ angle deviation. $60^{\circ}$ and $90^{\circ}$ away from the MFD exhibited similar properties. Fatigue strength exponents showed values in a range between $b=-0.045$ and $b=-0.057$ indicating similar fatigue performance degradation compared to GCL-4H samples. Considering the cross-sectional cuts in Fig. 5, it is obvious 

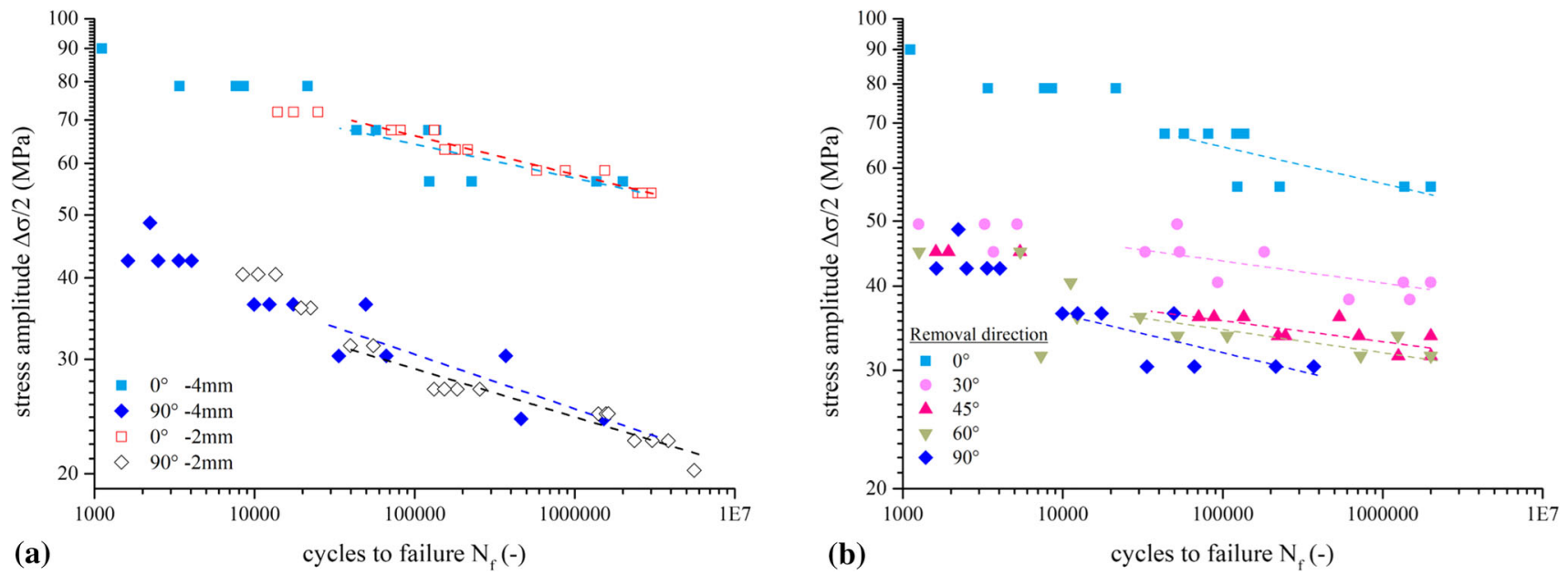

Fig. 9 Results of fatigue tests tested at $f=10 \mathrm{~Hz}, R=0.1$ and $T=R T$ with $\mathrm{SSx}^{\circ}$ samples. (a) Influence of specimen thickness and (b) influence of different removal directions

that except for $\mathrm{SS}^{\circ}$ no layer exhibits a favorable fiber orientation with respect to the applied load. In fact, the fibers are distributed randomly with elliptical shapes offering plenty potential damage sites. These heterogeneous microstructures could also be an explanation for the inherent strong scattering of the test results. As a possible consequence, fatigue strength exponent values range from -0.032 to -0.073 . The most pronounced fatigue life degradation rates were obtained for $0^{\circ}$ and $90^{\circ}$ samples, most likely reasoned by high loads for $\mathrm{SS}^{\circ}$ and large number of potential damage sites for $\mathrm{SS}^{\circ}$.

\subsection{Fatigue Damage Investigation}

Failure mechanisms during fatigue were studied investigating fracture surfaces via scanning electron microscopy. Regarding the damage course of fiber reinforced plastics, first cracks form over the entire sample volume. As soon as the micro crack concentration reaches a critical value, damage propagates continuously and reduces the stress-bearing area. Characteristic for this region is a high amount of dissipated heat in the form of a ductile fracture appearance. This damage phase of density increase can be described as stable crack propagation (Ref 20). When asking the question where initial cracks do initiate, it has to be considered whether cracks start as a consequence of stress concentration at fiber ends of fibers lying parallel to the loading direction or at fibers unfavorable orientated toward the axis of load. Generally, the weakest microstructural location in combination with the local stress creates a potential spot for crack initiation. A first evaluation can be done by using the wellestablished model of perpendicular and equidistantly oriented fibers lying in a strained matrix.

As soon as unfavorable orientated fibers in the core region are loaded, regions with high as well as low fiber concentrations have to be strained by the same amount. Caused by the higher Young's modulus of the fibers compared to the matrix, regions of high fiber content in the matrix polymer are deformed much more than in adjacent matrix-enriched regions in order to achieve strain equilibrium. Consequently, the deformation capacity of the matrix material is reached first in the regions of highest fiber content, so that the onset of fracture will take place there. However, it has to be noted that the shell layers are favorable for initial damage for directly injectionmolded LS. Bellenger et al. (Ref 10) investigated fatigue properties of short fiber-reinforced Polyamide standardized dog bone specimens and observed initial damage sites at fiber ends close to sample edges, where the stress concentration is highest. In contrast to an initial crack formation at the specimen edges, studies by Moosbrugger et al. (Ref 21) observed initial cracks forming in the core region of short fiber-reinforced polyamide tubular specimens. In studies of Szakács and Mészáros (Ref 22) the fatigue damage behavior of carbon fiber reinforced polyamide was assumed to take place over the whole specimen volume initially starting at fiber ends followed by void creation and a rigid, catastrophic breakage. To cover this, they divided the emerging fracture surface in a rigid and in a soft part in which the soft part is an indicator for stable crack propagation.

Fracture surfaces of $\mathrm{LS}$ and $\mathrm{SS}^{\circ}$ are illustrated in Fig. 10 and compared in terms of their fatigue damage characteristics. SEM micrographs in Fig. 10(a), (d) provide an overview of the fracture surface of LS after $2.42 \times 10^{6}$ cycles and $\mathrm{SS} 0^{\circ}$ after $1.37 \times 10^{6}$ cycles with the corresponding stresses of $\Delta \sigma /$ $2=68 \mathrm{MPa}$ and $\Delta \sigma / 2=56 \mathrm{MPa}$, respectively. The different layers described in Fig. 4 can clearly be distinguished, while the surface exhibits a change in fracture appearance. The inside of the dashed blue lines indicate areas of ductile matrix behavior. The outer region shows brittle fracture. Both, the degree of crazing and tearing, as well as the amount of fiber pull-out and debonding changes within the different regions. Details taken from fracture surfaces always revealed a clear transition from stable to unstable crack propagation. Stable crack propagation regions exhibited characteristic features for ductile matrix behavior: matrix yielding appearing in teared matrix filaments (Ref 23), as it can be seen in the micrographs (b), (e) and (f). Additionally, fiber surfaces free of matrix residues indicate fiber/matrix debonding. Unstable crack propagation covers characteristic features of brittle matrix behavior: fiber pull-out with undeformed hole edges and flat, insular matrix areas. This type of fracture occurrence can be found outside the dashed blue lines and is illustrated in Fig. 10(c) exemplary. Since the areas with the highest degree of ductility are the most-probable crack nucleation sites, it is notable that fatigue crack initiation occurred in the shell regions 

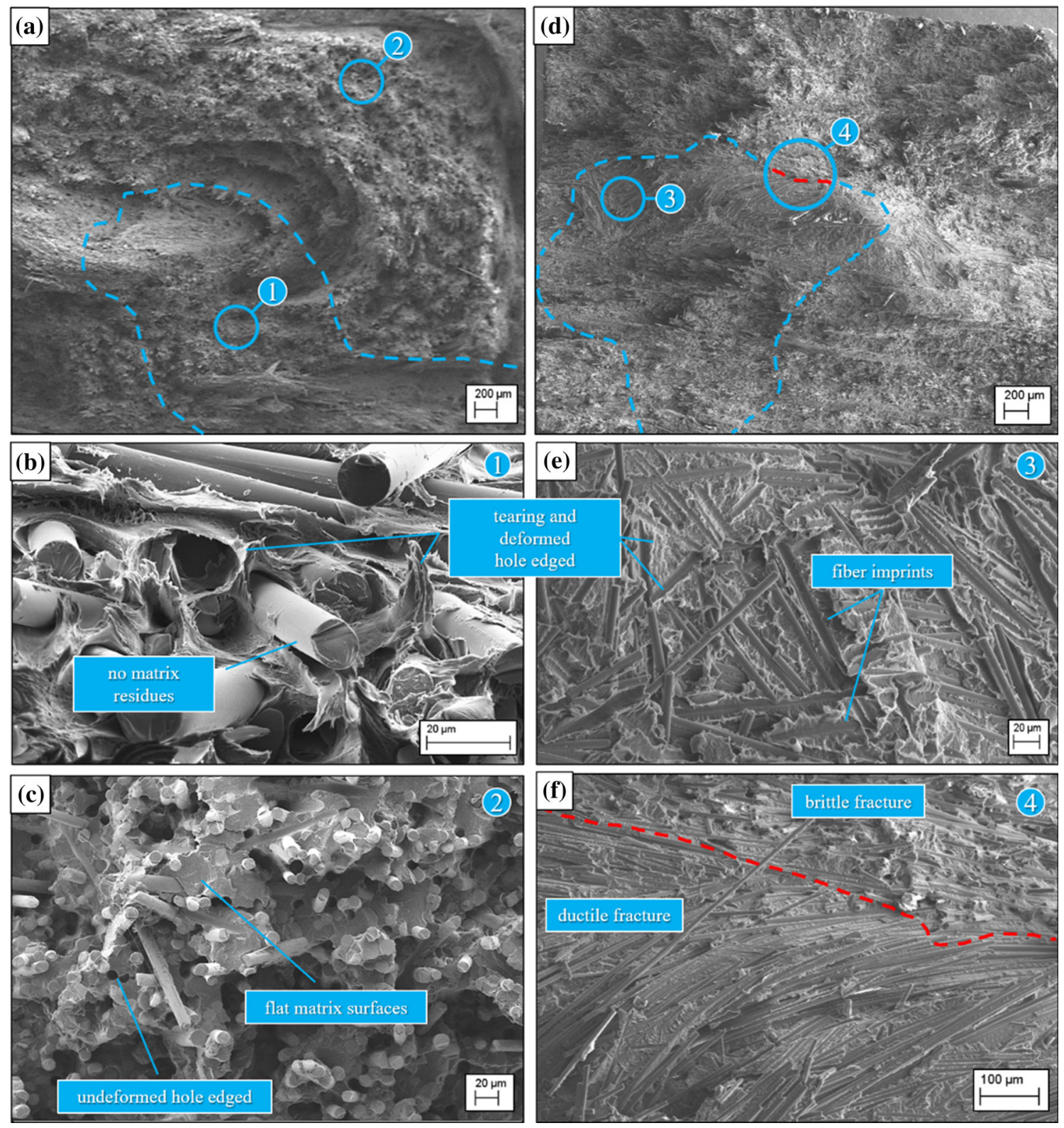

Fig. 10 SEM micrographs of fatigue fracture surfaces for LS (a-c) and for $\mathrm{SS}^{\circ}$ (d-f). Fracture surface overviews of (a) LS after $2.42 \times 10^{6}$ cycles (d) SS0 ${ }^{\circ}$ after $1.37 \times 10^{6}$ cycles. The inside of the dashed zones represent the areas of ductile fracture. Micrographs (b) and (c) illustrate typical appearances of ductile and brittle matrix behavior of LS. Crazed and teared matrix occurrence in the core region can be seen in picture (e) and (f)

of LS. Evaluating the framed areas for $\mathrm{LS}$ and $\mathrm{SS}^{\circ}$, it is striking that initial cracks occur in the core region for SSs. It can be taken from the inside of the dashed line in Fig. 10(d) that most of the ductile fractured areas are in inside regions. The highest degree of crazed and teared matrix filaments in the form of fiber/matrix debonding support the conclusion of fatigue crack initiation in the specimen core layers at unfavorable orientated fibers toward the axis of load. Reasons for this assumption are many fiber imprints on the surface from fibers lying perpendicular to the load axis (Fig. 10(e)-(f). Considering these observations, it is notable that except for directly injection-molded LS exhibiting high shell layer fractions, initial damage occurs in the core regions for milled SSs.

\section{Conclusion}

Quasi static and fatigue properties with respect to the prevalent microstructure of a $40 \mathrm{wt} \% \%$ long carbon fiber reinforced polyamide were investigated. Independent on the manufacturing process or sample thickness the specimens revealed a three-layered structure. The shell layer thickness remained constant, while the width of the core region increases with increasing specimen thickness. For standardized dog bone specimens vacuum voids were found in central regions located at fiber rich areas. Despite of changing injection molding process parameters such as back pressure to a maximum level an effect on the void content was not measurable. 
By investigating the longitudinal and transversal tensile property development as a function of the specimen thickness a crossing point was found for the Young's Modulus and tensile strength. This result suggests quasi-isotropic behavior at around $5 \mathrm{~mm}$ plate thickness.

For different removal directions relative to the axis of melt flow for both tensile and flexural properties a decrease for higher angle deviations starting from $0^{\circ}$ was observed. These changes are closely related to more unfavorable fiber orientation patterns in both the shell layer and the core layer.

Fatigue tests performed with dog bone specimens at different stress ratios yield the result that fully reversed loading conditions lead to the best fatigue performance with the lowest fatigue damaging rate. Weakest performance was found for $R=0.5$. The approach of the Basquin equation proved to be well fitting.

Examinations of the influence of specimen thickness, S-Ncurves for $2 \mathrm{~mm}$ and $4 \mathrm{~mm}$ thick samples milled at $0^{\circ}$ and $90^{\circ}$ to the axis of melt flow indicated no difference in fatigue resistance.

For a constant thickness of $4 \mathrm{~mm}$ the effect of different removal directions from a plate $\left(0^{\circ}, 30^{\circ} 45^{\circ}\right.$, and $\left.90^{\circ}\right)$ on the fatigue life came to the result that the fatigue performance is sensitive upon the loading axis relative to the axis of melt flow. Best properties were obtained $0^{\circ}$ to the melt flow direction followed by a constant decrease toward the $90^{\circ}$ orientated samples.

Fatigued fracture surfaces exhibited areas of brittle and ductile matrix behavior. Details taken from fracture surfaces revealed a clear transition from stable to unstable crack propagation. Stable crack propagation regions exhibited characteristic features for ductile matrix behavior: matrix yielding appearing in teared matrix filaments. Unstable crack propagation covers characteristic features of brittle matrix behavior: fiber pull-out with undeformed hole edges and flat, insular matrix areas.

\section{Acknowledgments}

The work is funded by the Ministry for Science and Culture under the LightConnect program, project VWZN3223. We gratefully thank EMS-Chemie AG for providing the raw materials and Arburg $\mathrm{GmbH} \& \mathrm{Co} \mathrm{KG}$ for providing the injection molding machine. Further, we thank our partners from the industry Volkswagen Osnabrück GmbH and BOGE Elastmetall GmbH for the support.

\section{Funding}

Open Access funding enabled and organized by Projekt DEAL.

\section{Data Availability}

Raw data were generated exclusively in laboratories of the Hochschule Osnabrück. Derived data supporting the findings of this study are available from the corresponding author on request.
Open Access This article is licensed under a Creative Commons Attribution 4.0 International License, which permits use, sharing, adaptation, distribution and reproduction in any medium or format, as long as you give appropriate credit to the original author(s) and the source, provide a link to the Creative Commons licence, and indicate if changes were made. The images or other third party material in this article are included in the article's Creative Commons licence, unless indicated otherwise in a credit line to the material. If material is not included in the article's Creative Commons licence and your intended use is not permitted by statutory regulation or exceeds the permitted use, you will need to obtain permission directly from the copyright holder. To view a copy of this licence, visit http://creativecommons.org/licenses/by/4.0/.

\section{References}

1. J. Markarian, Long Fibre Reinforced Thermoplastics continue growth in automotive Plastics, Addit. Compd., 2007, 9, p 20-24

2. Markets and Market (2020): Thermoplastic Composites Market by Resin Type (Polypropylene, Polyamide, Polyetheretherketone, Hybrid), Fiber Type (Glass, Carbon, Mineral), Product Type (SFT, LFT, CFT, GMT), End-Use Industry, and Region: Global Forecast to 2025, Report CH3527

3. J. Thomason, The Influence of Fiber Length and Concentration on the Properties of Glass Fiber Reinforced Polypropylene: 7 Interface Strength and Fiber Strain in injection molded Long Fiber PP at high Fiber Content, Compos. Part A Appl. Sci. Manuf., 2007, 38, p 210-216

4. Y. Zhou and P.K. Mallick, Fatigue Performance of an Injection-Molded Short E-Glass Fiber-Reinforced Polyamide 6,6. I. Effects of Orientation Holes and Weld Line, Polym. Compos., 2006, 27, p 230-237

5. J. Brunbauer, A. Mösenbacher, C. Guster and G. Pinter, Fundamental Influences on Quasi static and Cyclic Material Behavior of Short Glass Fiber Reinforced Polyamide Illustrated on Microscopic Scale, J. Appl. Polym. Sci., 2014 https://doi.org/10.1002/app.40842

6. E. Wollan, Glass \& Carbon Fiber Reinforcement Combine in Hybrid Long Fiber Thermoplastic Composites to Bridge Price \& Performance Gap, Reinf. Plast., 2017, 61, p 55-57

7. A. Bernasconi and P. Davoli, Effect of Fibre Orientation on the Fatigue behavior of a Short Glass Fiber Reinforced Polyamide, Int. J. Fatigue, 2007, 29, p 200-208

8. D. Grove and H. Kim, Fatigue Behavior of Long and Short Glass Reinforced Thermoplastics, J. Mater. Manuf., 1995, 104, p 450-456

9. A. Goel, K.K. Chawla, U.K. Vaidya, N. Chawla and M. Koopman, Characterization of Fatigue Behavior of Long Fiber Reinforced Thermoplastic (LFT) Composites, Mater. Charact., 2009, 60, p 537544

10. V. Bellenger, A. Tcharkhtchi and Ph. Castaing, Thermal and Mechanical Fatigue of a PA66/Glass Fibers Composite Material, Int. J. Fatigue, 2006, 28, p 1348-1352

11. M. Bondy, W. Rodgers and W. Altenhof, Tensile Fatigue Characterization of Polyamide 66/Carbon Fiber Direct/in-Line Compounded Long Fiber Thermoplastic Composites, Compos. Part B Eng., 2019 h ttps://doi.org/10.1016/j.compositesb.2019.106984

12. F.N. Cogswell, Thermoplastic Aromatic Polymer Composites: A Study of the Structure, Processing and Properties of Carbon Fibre Reinforced Polyetheretherketone and Related Materials, Elsevier, 2013

13. N. Graupner, G. Ziegmann, H. Enzler, J. Müssig, K.K. Albrecht and K. Albrecht, Influence of Reprocessing on Fiber Length Distribution, Tensile Strength and Impact Strength of Injection Moulded Cellulose 
Fiber-Reinforced Polylactide (PLA) Composites, Exp. Polym. Lett., 2016, 10(8), p 647-663

14. K.S. Kumar, V. Patel, A. Tyagi, N. Bhatnagar and A.K. Ghosh, Injection Molding of Long Fiber Reinforced Thermoplastic Composites, Int. Polym. Process., 2009, 24, p 17-22

15. H. Ning, N. Lu, A.A. Hassen, K. Chawla, M. Selim and S. Pillay, A Review of Long Fibre Thermoplastic (LFT) Composites, Int. Mater. Rev., 2020, 65(3), p 164-188

16. O.H. Basquin, The Exponential Law of Endurance Tests, Am. Soc. Test. Mater. Proc., 1910, 10, p 625-630

17. B. Harris, A Historical Review of the Fatigue Behavior of FibreReinforced Plastics, Fatigue in Composites: Science and Technology of the Fatigue Response of Fibre-Reinforced Plastics. B. Harris Ed., Elsevier, 2003

18. P. Alam, D. Mamlis, C. Robert, C. Floreani and C.O. Brádaigh, The Fatigue of Carbon Fibre Reinforced Plastics: A Review, Compos. Part B Eng., 2019, 166, p 555

19. J.J. Horst, Influence of Fiber Orientation of Short Glass Fiber Reinforced Polyamide, Universität Delft, Delft, Habilitation, 1997
20. E. Belmonte, M. De Monte, C.-J. Hoffmann and M. Quaresimin, Damage Mechanisms in a Short Glass Fiber Reinforced Polyamide under Fatigue Loading, Int. J. Fatigue, 2017, 94, p 145-157

21. E. Moosbrugger, M. De Monte, K. Jaschek and M. Quaresimin, Multiaxial Fatigue of a Short Glass Fibre Reinforced Polyamide 6.6: Fatigue and Fracture Behavior, Int. J. Fatigue, 2010, 32, p 17-28

22. J. Szakács and L. Mészáros, Effect of Fiber Contents on Fatigue Behavior of Injection Molded Polyamide 6 Matrix Composites, Period. Polytech. Mech. Eng., 2017, 61(1), p 74-78

23. H. Rolland, N. Saintier, I. Raphael, N. Lenoir, A. King and G. Robert, Fatigue Damagemechanisms of Short Fiber Reinforced PA66 as Observed by In-Situ Synchrotron X-ray Microtomography, Compos. Part B, 2018 https://doi.org/10.1016/j.compositesb.2017.12.051

Publisher's Note Springer Nature remains neutral with regard to jurisdictional claims in published maps and institutional affiliations. 